EÉSTI NSV TEADUSTE AKADEEMIA TOIMETISED. 31. KOIDË FOOSIKA * MATEMAATIKA. 1982, NR. 2

ИЗВЕСТИЯ АКАДЕМИИ НАУК ЭСТОНСКОП ССР. ТОМ 31 ФИЗНКА * МАТЕМАТИКА. 1982, № 2

Л. Л. ВАЛКУНАС, Э. ГАИЖАУСКАС,

УдК $535.34: 621.373 .8$ Ш. П. КУДЖМАУСКАС

\title{
ТЕОРЕТИЧЕСКОЕ ИССЛЕДОВАНИЕ АБСОРБЦИОННЫХ ИЗМЕНЕНИЙ В РЕАКЦИОННЫХ ЦЕНТРАХ ФОТОСИНТЕЗИРУЮЩИХ БАКТЕРИИ
}

Первичные процессы, протекающие в реакционных центрах (РЦ) фотосинтезирующих систем, изучаются в последнее время методами пикосекундной лазерной спектроскопии. В случае использования при этом ультракоротких лазерных импульсов для возбуждения и зондирования на разных частотах сопоставление полученных результатов с теоретическими расчетами дает возможность, в принципе, определять как кинетические параметры системы, так и энергетические состояния, участвующие в переносе электрона в РЦ. Однако в широких пределах изменения интенсивности и длительности возбуждающих импульсов лазера могут проявить себя нелинейные и когерентные эффекты взаимодействия РЦ со светом, что необходимо учитывать при их теоретическом описании.

В настоящем сообщении проанализированы первичные стадии физических превращений в РЦ фотосинтезирующих бактерий, возбужденных ультракоротким импульсом лазера. Энергетическая модель РЦ (рис. 1) заимствована нами из [ $\left.{ }^{1}\right]$, где она построена на основе многочисленных экспериментов по поглощению сгета РЦ, ЭПР-спектроскопии и др. Она содержит нижайшие синглетные переходы димера и мономера бактериохлорофилла, $P_{F}$-состояние, а также состояние, в котором электрон переходит на первичный акцептор хинонной природы $\left(P^{+} Q^{-}\right)$. Әволю-

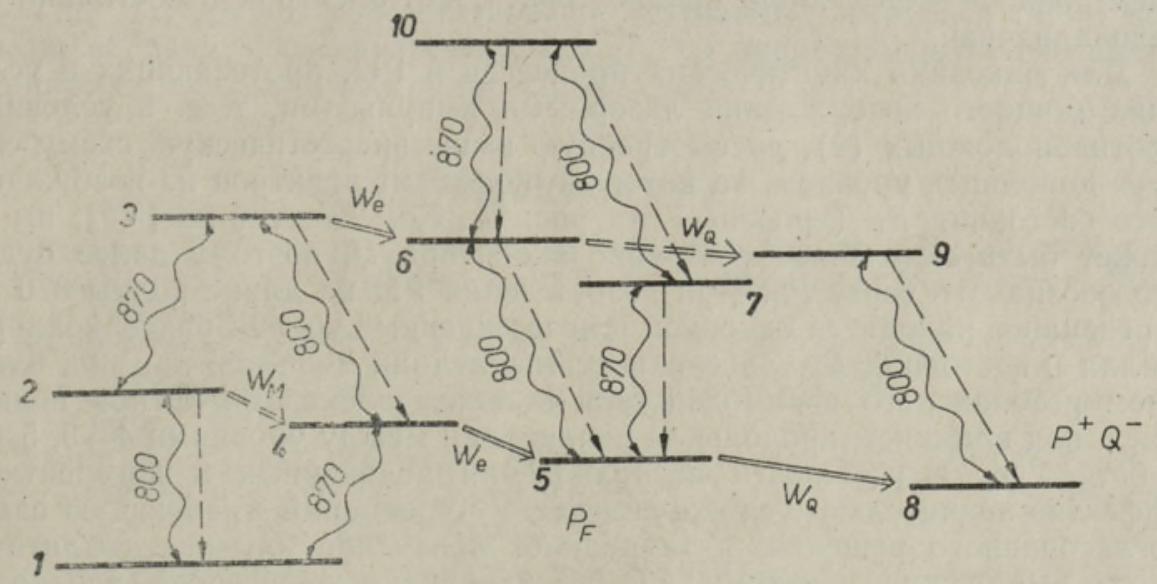

Рис. 1. Схема энергетических уровней РЦ: 1 - основное состояние системы; 2 и 4 - возбужденные уровни P800 и Р870; 3 - их совместное возбуждение; $5-7,10$ - основной и возбужденные уровни состояния $P_{F} ; 8$ и 9 - основное и возбужденное состояния $P+Q^{-} . W_{e}, W_{Q}$ и $W_{M}-$ скорости продольной релаксации. 
ция РЦ, взаимодействующих с полем электромагниитной волны, опйсывалась нами уравнениями для элементов матрицы плотности. Последние решались совместно с уравнениями Максвелла. Такая самосогласованная система уравнений учитывает возможность влияния среды на излучение при высоких уровнях возбуждения.

В условиях слабого возбуждения РЦ, когда время фазовой релаксации $T_{2}$, коэффициент поглощения $\sigma_{m n}$, интенсивность возбуждающего импульса $I_{0}$ и его длительность $\tau_{0}$ удовлетворяют неравенству

$$
\sigma_{m n} I_{0} \tau_{0}^{-1} \ll T_{2}^{-1},
$$

нами получены аналитические выражения для заселенностей энергетических уровней РЦ. При возбуждении РЦ на длине волны перехода димера и при зондировании мономера бактериохлорофилла изменение оптической плотности образца $\triangle A$ представляется в виде

$$
\begin{gathered}
\Delta A=(\ln 10)^{-1} \ln \left(\varrho_{11}(d, t) / \varrho_{11}(0, t)\right) \sigma_{41}^{-1} X \\
\times\left\{\sigma_{21}-\sigma_{98} f\left(\sigma_{98}-\sigma_{65}\right) \exp \left(-W_{x} t\right)\right\},
\end{gathered}
$$

где

$$
\mathrm{Q}_{11}(x, t)=\exp \left(\sigma_{41} \varepsilon_{0}^{-1} N_{\mathrm{PL}} x\right)\left\{\exp \left(\sigma_{41} \varepsilon_{0}^{-1} N_{\mathrm{PL}} x\right)+\exp \left[\sigma_{41} \int_{-\infty}^{t} I(t) d t\right]-1\right\}^{-1}
$$

- заселенность основного состояния системы РЦ, $N_{\mathrm{pц}}$ - концентрация РЦ, $I(t)$ - интенсивность возбуждения. Сопоставление теоретических расчетов (см. $\left.\left[{ }^{2}\right]\right)$ по формуле (2) с серией экспериментальных кривых $\left[{ }^{3,4}\right]$ позволяет определить спектры поглощения $\sigma_{65}(\lambda), \sigma_{98}(\lambda)$. Оказывается, что полоса поглощения мономера бактериохлорофилла одинаково смещается как в состоянии $P_{F}$, так и в состоянии $P+Q^{-}$, а интенсивность поглощения почти не изменяется. Это говорит о том, что электрон на мономере бактериохлорофилла или вб́лизи него не локализуется. Штарковское смещение обусловлено, вероятно, электрическим полем ионизованного первичного фотодонора, расположенного вблизи мономера бактериохлорофилла. Поскольку отсутствие изменений в полосе первого синглетного перехода бактериофеофитина при линейном возбуждении РЦ $\left[{ }^{5}\right]$ также свидетельствует против локализации на нем электрона, остается лишь предположить, что электрон в состоянии $P_{F}$ делокализован.

Для описания кинетических процессов в РЦ, протекающих в условиях мощного возбуждения лазерными импульсами, т. е. в условиях, противоположных (1), рассмотренную выше энергетическую схему следует дополнить уровнем, на который переходит электрон из возбужденного состояния $P_{F}$ (уровень 7 на рис. 1). Как следует из $\left[{ }^{3-5}\right]$, им не может быть окисленное состояние акцептора $Q$, поэтому далее будем его обозначать через $P_{G}$. При возбуждении РЦ на длине волны $870 \mathrm{HM}$ в резонансе находятся основной и возбужденные уровни бактериохлорофилла и состояния $P_{F}$. Заселенность последних меняется как под влиянием резонансного взаимодействия с полем электромагнитной волны, так и под влиянием продольной релаксации между уровнями $4 \rightarrow 5,5 \rightarrow 8$, $7 \rightarrow P_{G}$. Учет возможности возникновения нелинейных и когерентных эффектов значительно усложняет систему уравнений, требующую самосогласованного решения, и затрудняет получение для нее аналитического выражения. Поэтому анализ изменения оптической плотности образца проводился нами численными методами.

На рис. 2 показаны результаты вычислений для разных площадей импульса

$$
\Psi_{\lambda}=\mu_{n m} \hbar-1 \int_{-\infty}^{\infty} E(t) d t
$$


Рис. 2. Изменение оптической плотности в случае зондирования Р800 при нелинейном возбуждении на длине волны 870 нм: $\Psi_{870}=3 \pi(1), 12 \pi \quad(2$, 3); $\tau_{0} / T_{2}=25$ (2) и 10 (3).

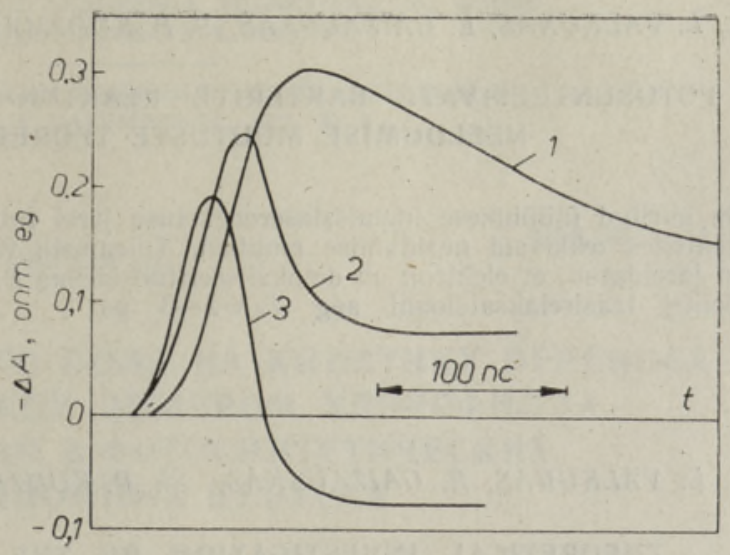

где $\mu_{n m}$ - диполный момент перехода, $E(t)$ - напряженность возбуждающей волны, и для разных параметров $T_{2}$. Ряд констант определен из экспериментальных результатов, полученных в линейной области.

Результаты показывают, что когерентные эффекты, действительно, имеют место и, следовательно, должны учитываться в экспериментах по пикосекундной спектроскопии РЦ. Так, на рис. 2 видны значительные нелинейные изменения оптической плотности образца для соотношения $\tau_{0} / T_{2}=10$ при мощном возбуждении РЦ $\left(\Psi_{\lambda} \gg \pi\right)$. И хотя при $\tau_{0} / T_{2} \gg 1$ нелинейные эффекты довольно сильно подавляются, необходимость учета когерентных эффектов остается.

Представляет интерес возможность определения времени фазовой релаксации в системе РЦ по нелинейным изменениям оптической плотности образца в зависимости от интенсивности возбуждающего импульса. Так, например, в $\left[{ }^{3,4}\right]$ абсорбционные изменения в полосе 802 нм определены по резонансному возбуждению фотодонора. Кинетика абсорбционных изменений в указанных работах качественно меняется в случае превышения порогового уровня, равного $\simeq 10^{16}$ фотон/ $\mathrm{cm}^{2}$. Если матричный элемент дипольного перехода $\mu_{41}$ положить равным $5 D$, то возбуждающему импульсу такой интенсивности (длительность $\left.\tau_{0}=30 n c\right)$ будет соответствовать «площадь» импульса $\Psi=1,5 \pi$. Таким образом, согласуя результаты $[3,4]$ с приведенными нами вычислениями, получаем время релаксации $T_{2}=2-3 \mathrm{nc}$.

\section{ЛИТЕРАТУ РА}

1. В а лкунас Л., Куджм а ускас Ш., Препринт ИФІ-79, Внльнюс, 1979.

2. Gaižauskas, E., Kudžmauskas, S., Valkunas, L., Preprint IPI-81, Vilnius, 1981.

3. Ахманов С. А., Борисов А. Ю., Козловский В. С., Разживин А. П., Гадонас Р. А., Данелюс Р. В., Пискарскас А. С., В кн.: Нелинейная оптика, Tp. VI Вавиловской конференцин, ч. 2, Новосибирск, «Наука», 1979 , c. $74-84$.

4. Akhmanov, S. A., B or is ov, A. Yu., D a nielius, R. V., Gadonas, R. A., Kozlowski, V. S., Piskarskas, A. S., Razjivin, A. P., In: Laser Spectroscopy IV (ed. H. Walter, K. W. Rothe), Springer-Verlag, Berlin-Heidelberg-New York, 1979, p. 387-398.

5. Akhmanov, S. A., B orisov, A. Yu., Darielius, R. V., Gadonas, R. A., Kozlowski, V. S., Piskarskas, A. S., Razjivin, A. P., Shuv a 1 o v, V. A., FEBS Lett., 114, № 1, 149-152 (1980).

Институт физики

Академии наук Литовской ССР 


\section{FOTOSUNTEESIVATE BAKTERITE REAKTSIOONITSENTRITES ILMNEVATE NEELDUMISE MUUTUSTE TEOREETILINE UURIMUS}

On uuritud ülilühikese impulsslaserergastuse järel fotosünteesivate bakterite reaktsioonitsentrites tekkivaid neeldumise muutusi. Tulemuste võrdlemisest eksperimendiandmetega on järeldatud, et elektron on delokaliseeritud olekus $P_{F}$, samuti on määratud reaktsioonitsentri faasirelaksatsiooni aeg $T_{2} \approx 2-3$ ps.

L. L. VALKUNAS, E. GAIZAUSKAS, Sh. P. KUDZMAUSKAS

\section{THEORETICAL INVESTIGATION OF THE ABSORPTION CHANGES IN THE REACTION CENTRES OF PHOTOSYNTHETIC BACTERIA}

We have carried out a theoretical investigation of the absorption changes in the reaction centres (RC) of photosynthetic bacteria under the ultra-short laser pulse excitation. In the case of a weak excitation of a bacteriochlorophyll dimer $(870 \mathrm{~nm})$ and probing at the $800 \mathrm{~nm}$-wavelength, the change of the optical density $(\Delta A)$ is expressed analytically. In the case of a powerful excitation when coherent and nonlinear effects must be included into consideration, the analysis of $\Delta A$ is carried out numerically. Comparing these results with those of known experiments it is concluded that the electron is delocalized in $P_{F}$ state. The phase relaxation time in the RC system is estimated to be $2-3$ ps. 